\title{
Keynote Speaker
}

\section{Mimicking Nature for Reliable and Secure Computer Systems}

\author{
Elhadj Benkhelifa, ${ }^{a, *}$ \\ ${ }^{\mathrm{a} S t a f f o r d s h i r e ~ U n i v e r s i t y, ~ U n i t e d ~ K i n g d o m ~}$ \\ *Corresponding Author: elhadj.benkhelifa@googlemail.com
}

\begin{abstract}
This talk will shed lights on the concept of Artificial Life (A-Life) and the latest advances in the field and future projections. I will present a summary of my research interests in this area, illustrated by cutting edge projects. The main essence of these projects is biologically and nature inspired for self-organizing, self-healing and self-adaptive computer system for enhanced reliability and resilience. These unconventional solutions are gaining a growing interest among researchers in many applications at the time when traditional approaches proved inefficient, especially with the ever increasing hardware faults and threat of cyber-attacks in critical complex infrastructures such as cloud platforms.
\end{abstract}

